\title{
Acoustic and Perceptual Consequences of Clear and Loud Speech
}

\author{
Kris Tjaden $^{a}$ Emily Richards ${ }^{a} \quad$ Christina Kuo $^{a, c} \quad$ Greg Wilding $^{b}$ Joan Sussman ${ }^{a}$ \\ ${ }^{a}$ Department of Communicative Disorders and Sciences and ${ }^{b}$ Department of Biostatistics, University at Buffalo, \\ Buffalo, N.Y., 'Department of Communication Sciences and Disorders, James Madison University, Harrisonburg, Va., \\ USA
}

\section{Key Words}

F2 slope · Clear speech · Loud speech · Intelligibility

\begin{abstract}
Objective: Several issues concerning F2 slope in dysarthria were addressed by obtaining speech acoustic measures and judgments of intelligibility for sentences produced in Habitual, Clear and Loud conditions by speakers with Parkinson's disease (PD) and healthy controls. Patients and Methods: Acoustic measures of average and maximum F2 slope for diphthongs, duration and intensity were obtained. Listeners judged intelligibility using a visual analog scale. Differences in measures among groups and conditions as well as relationships among measures were examined. Results: Average and maximum F2 slope metrics were strongly correlated, but only average F2 slope consistently differed among groups and conditions, with shallower slopes for the PD group and steeper slopes for Clear speech versus Habitual and Loud. Clear and Loud speech were also characterized by lengthened durations, increased intensity and improved intelligibility versus Habitual. F2 slope and intensity were unrelated, and F2 slope was a significant predictor of intelligibility. Conclusion: Average diphthong F2 slope was more sensitive than maximum F2 slope to articulatory mechanism involvement in mild dysarthria in PD. F2 slope holds promise as an objective measure of treatment-related changes in the articulatory mechanism for therapeutic techniques that focus on articulation.

๑) 2014 S. Karger AG, Basel
\end{abstract}

Portions of this study were presented at the Fall 2011 Meeting of the Acoustical Society of America as well as the 2010 Convention of the American Speech-Language and Hearing Association.

\section{Introduction}

The slope of the second formant (F2) frequency is an acoustic measure reflecting rate of vocal tract shape change [1]. Shallower slopes also are associated with slower lingual movement speeds [2]. Compared to healthy talkers, reduced F2 slopes have been reported for a variety of dysarthrias and neurological diagnoses, including Parkinson's disease (PD) - the clinical population of interest to the current study [see reviews in 1-5]. Research further suggests that F2 slope measures do not differ for dysarthrias of different etiologies or types $[4,5]$. Finally, F2 slope is sensitive to dysarthria severity such that speakers with more severe dysarthria or relatively poorer intelligibility have shallower F2 slopes compared to speakers with less severe dysarthria or relatively better intelligibility $[2,4,5]$.

Although F2 slope in dysarthria has been studied a fair amount, several issues deserve additional attention. One of these issues is the utility of average F2 slope metrics, as reported in the studies reviewed in the preceding paragraph, versus extreme F2 slope metrics for characterizing articulatory behavior in dysarthria. By way of background, F2 slope is typically quantified as transition extent divided by transition duration, with transition onset

Kris Tjaden, $\mathrm{PhD}$

Department of Communicative Disorders and Sciences, University at Buffalo 122 Cary Hall, 3435 Main Street

Buffalo, NY 14214 (USA)

E-Mail Tjaden@buffalo.edu

\section{KARGER}

E-Mail karger@karger.com www.karger.com/fpl 
and offset identified using the $20 \mathrm{~Hz} / 20 \mathrm{~ms}$ rule [6]. The traditional slope measure thus reflects overall or average rate of spectral change during the operationally defined transition interval. F2 slope also may be computed on a point-by-point basis from a linear predictive coding (LPC)-generated F2 trajectory to yield a time history of instantaneous slope values. The transition interval is still identified using the $20 \mathrm{~Hz} / 20 \mathrm{~ms}$ rule and instantaneous slope values are averaged over the transition interval to provide an overall or average F2 slope metric which correlates strongly with the traditional transition extent/ transition duration measure $[2,7]$. This approach also allows extreme instantaneous slope values, such as maxima, to be identified. A recent study of multiple sclerosis further concluded that extreme F2 slope measures were more sensitive to mild dysarthria than average slope measures [8]. Only one dysarthria study has reported extreme F2 slope metrics, however, and additional studies obviously are needed before drawing strong conclusions regarding the utility of extreme F2 slope metrics in mild dysarthria. Indeed, there is likely some redundancy between average and extreme slope measures owing to their part-whole relationship, although the relationship has not been empirically evaluated.

F2 slope also shows strong potential as an objective measure of treatment-related changes in the articulatory mechanism for individuals with PD [1], yet few treatment-related studies of PD have reported F2 slope measures. Moreover, results of these studies which have employed formal training programs such as the Lee Silverman Voice Treatment ${ }^{\circledR}$ (LSVT) as well as stimulation are equivocal $[9,10]$. An increased vocal intensity is probably the most widely used treatment technique for PD, but clear speech is another global therapy technique that shows promise for addressing the speech impairment in PD as well as for enhancing intelligibility [11]. Global techniques span the time domain of an utterance and have the potential to simultaneously impact multiple speech subsystems [11]. In this manner, although an increased vocal intensity focuses on modifying respiratory-phonatory behavior, adjustments in segmental articulatory behavior may occur. Similarly, clear speech focuses on exaggerated articulation, but respiratory-phonatory adjustments may also accompany clear speech. Comparative studies are critical for determining the relative advantages of therapeutic techniques, but studies comparing an increased intensity and clear speech in PD are lacking.

In conclusion, the present study sought to address several issues concerning F2 slope in dysarthria. First, the utility of an extreme or maximum F2 slope measure for characterizing articulatory behavior in dysarthria above and beyond the traditional, average or overall F2 slope measure is not well understood. The sensitivity of F2 slope to articulatory changes elicited by different global dysarthria therapy techniques also is not well established. The current study sought to advance understanding of these issues by comparing the impact of clear speech and an increased vocal intensity on average and maximum F2 slope metrics for diphthongs produced by speakers with mild dysarthria secondary to PD as well as healthy controls. The strength of the relationship between average and maximum F2 slope metrics was quantified as was the relationship between F2 slope and vocal intensity. Our interest in the relationship between F2 slope and vocal intensity was motivated by statements in the dysarthria literature concerning the potential 'spreading effect' of an increased vocal effort to the articulatory mechanism $[12,13]$, although studies of neurologically normal speech have demonstrated the reverse - that articulatory adjustments impact phonatory behavior [e.g., 14, 15]. Given the potential for clear speech and an increased intensity to enhance intelligibility as well as long-standing interest in the relationship between F2 slope and intelligibility in dysarthria, we also investigated the predictive relationship of F2 slope to intelligibility for the PD group. Magnitude production was used to elicit an increased vocal intensity and clear speech. Although results are not directly comparable to studies employing training, studies investigating experimental manipulation of speech suggest the potential of intervention techniques $[11,16]$. Finally, although speakers with PD in the current study had mild dysarthria, even persons with mild dysarthria may benefit from treatment focused on an increased vocal intensity or clear speech $[11,16]$.

\section{Method}

Speakers

Thirteen speakers with idiopathic PD and 15 healthy controls who are part of a larger project were included for study [17]. The PD group was comprised of 7 men and 6 women $48-78$ years of age $($ mean $=68 ; \mathrm{SD}=10)$, and the control group was comprised of 8 men and 7 women $46-75$ years of age $($ mean $=61 ; S D=10)$. Participants were native speakers of American English, spoke with the Inland North dialect of western New York State and scored at least 26/30 on the Standardized Mini-Mental State [18]. No speaker used a hearing aid or had undergone neurosurgery. Two females with PD had completed LSVT more than 2 years prior to the study and one of these speakers was enrolled in a group, bimonthly LSVT 
refresher course. Speakers with PD were recorded $1 \mathrm{~h}$ prior to taking medication. All speakers were paid USD 10 per hour.

Table 1 describes the PD group. Sentence intelligibility scores for 10 student listeners as well as 3 speech-language pathologists' mean judgment of speech severity for the 'Grandfather Passage' were previously reported [17]. The operationally defined perceptual construct of speech severity aims to tap into prosodic adequacy and naturalness with values closer to 1.0 indicating more impairment and values closer to 0 indicating less impairment. Sentence intelligibility test scores [19] in table 1 for the PD group (mean $=89 ; \mathrm{SD}=3$ ) coupled with mid-range judgments of speech severity (mean $=0.54$; $\mathrm{SD}=0.21)$ are consistent with clinical descriptions of mild dysarthria [16]. For comparison, the mean sentence intelligibility test score for the control group was 93 (SD = $2)$, and mean speech severity was $0.26(\mathrm{SD}=0.14)$. The slightly reduced intelligibility and elevated speech severity scores for controls likely reflect the fact that speech samples were pooled across normal and disordered speakers for these analyses [17]. Speakers with PD were further noted to have reduced segmental precision and a breathy, monotonous voice.

\section{Experimental Speech Stimuli and Procedures}

Speakers were audio recorded in a sound-treated room reading 25 Harvard Psychoacoustic Sentences [20] in a variety of conditions. The Habitual, Clear and Loud conditions were of interest to the current study. Across 12 sentences, there were 10 occurrences of /aI/ and 4 occurrences of /or/ in stressed syllables of content words varying in sentence position and phonetic context. The acoustic signal was transduced using an AKG C410 head-mounted microphone positioned $10 \mathrm{~cm}$ and $45-50^{\circ}$ from the left oral angle. The signal was preamplified, low pass-filtered at $9.8 \mathrm{kHz}$ and digitized to computer hard disk at a sampling rate of $22 \mathrm{kHz}$ using TF32 [21]. A calibration tone also was recorded to allow for offline measurement of vocal intensity from the acoustic signal. For the Loud condition, participants were instructed to use a vocal intensity twice as loud as their typical speech. For the Clear condition, participants were instructed to say each sentence twice as clearly as their typical speech. Speakers were told to exaggerate the movements of their mouth and were instructed that this is how they might speak to someone in a noisy environment or to someone with a hearing loss. Speakers also were told that their speech might be slower and louder than usual. Instructions were modeled after those used in other clear speech studies [22]. For each speaker and condition, a unique ordering of sentences was recorded. Sentences were read first in the Habitual condition with the order of the remaining conditions randomized across speakers.

\section{Acoustic Analysis}

Acoustic measures were obtained using TF32. Measures of sentence-level sound pressure level (SPL) and articulatory rate served to document that the magnitude production paradigm elicited production differences among conditions. Diphthong durations, described in the following paragraph, further documented segment-level duration differences among conditions. Sentences first were segmented into runs using the combined waveform and wideband (300-400 Hz) spectrographic displays. A run was operationally defined as a stretch of speech bounded by silent periods between words of at least $200 \mathrm{~ms}$. Run onsets and offsets were identified using conventional acoustic criteria. Articulatory rate, in syllables per second, was determined by tallying the number of syl-
Table 1. Characteristics for participants with PD

\begin{tabular}{llllll}
\hline Subject code & Sex & Age & $\begin{array}{l}\text { Years after } \\
\text { diagnosis }\end{array}$ & $\begin{array}{l}\text { Sentence } \\
\text { intelligibility } \\
\text { score, \% }\end{array}$ & $\begin{array}{l}\text { Scaled speech } \\
\text { severity } \\
\text { 'Grandfather } \\
\text { Passage' }\end{array}$ \\
& & & & & 0.85 \\
PD 01 & F & 76 & 20 & 84 & 0.38 \\
PD 02 & F & 78 & 3 & 95 & 0.59 \\
PD 04 & F & 48 & 11 & 90 & 0.68 \\
PD 05 & F & 74 & 2 & 87 & 0.62 \\
PD 06 & F & 75 & 5 & 90 & 0.33 \\
PD 08 & F & 63 & 2 & 89 & 0.49 \\
PD 01 & M & 76 & 12 & 87 & 0.51 \\
PD 02 & M & 65 & 8 & 89 & 0.70 \\
PD 03 & M & 58 & 13 & 89 & 0.17 \\
PD 04 & M & 55 & 5 & 92 & 0.23 \\
PD 06 & M & 66 & 3 & 91 & 0.67 \\
PD 07 & M & 67 & 32 & 90 & 0.80 \\
PD 08 & M & 78 & 4 & 90 & $0.54 \pm 0.21$ \\
\hline Mean \pm SD & & $68 \pm 9$ & $9 \pm 8$ & $89 \pm 3$ & \\
\hline & & & & & \\
\hline
\end{tabular}

lables per run and dividing by run duration. Mean SPL for each run was obtained by using the root-mean-squared intensity trace of TF32. For each speaker and condition, SPL and rate measures were averaged across runs.

Diphthong onsets and offsets were identified and labeled from the combined waveform and wideband $(300-400 \mathrm{~Hz})$ spectrographic displays. Pitch-synchronous LPC tracks on a wideband (300-400 Hz bandwidth; 26 coefficients) spectrogram were generated and computer-generated tracking errors were manually corrected. Diphthong duration was calculated as the time between diphthong onset and offset. Instantaneous slope was computed for each point of the LPC F2 formant time history as the change in frequency $(\mathrm{Hz})$ divided by the change in time $(\mathrm{ms})$. Instantaneous slope values were subsequently used to identify the onset and offset of each major, rising F2 transition using operational criteria approximating the $20 \mathrm{~Hz} / 20 \mathrm{~ms}$ rule [6]. Maximum F2 slope was identified as the maximum instantaneous slope value of the F2 transition. Average F2 slope was obtained by averaging all instantaneous slope values during the operationally defined transition. For each speaker, condition and diphthong, segment durations and F2 slope measures were averaged across tokens.

\section{Perceptual Task}

Fifty listeners aged 18-30 years judged sentence intelligibility. Listeners were native speakers of standard American English, had at least a high school diploma, reported no history of speech, language, or hearing problems, passed a hearing screening at $20 \mathrm{~dB} \mathrm{HL}$ for octave frequencies from 250 to $8,000 \mathrm{~Hz}$ bilaterally and were unfamiliar with speech disorders. Listeners were paid USD 10 per hour.

For each speaker, a random selection of the same 10 Harvard Sentences produced in all conditions was studied to allow the perceptual task to be completed in a single session. To prevent ceiling effects, sentences were presented in multitalker babble, as is commonly done in the clear speech literature [20]. Sentences first were normalized for 
Table 2. Means and SDs for speech durations, vocal intensity and scaled sentence intelligibility

\begin{tabular}{llccc}
\hline Measure & Group & Habitual & Clear & Loud \\
\hline Articulatory rate, syllables/s & control & $3.7 \pm 0.5$ & $2.4 \pm 0.4$ & $3.3 \pm 0.6$ \\
& PD & $4.0 \pm 0.6$ & $3.1 \pm 0.7$ & $3.9 \pm 0.7$ \\
SPL, dB & control & $74 \pm 2.8$ & $79 \pm 4.4$ & $85 \pm 4.2$ \\
& PD & $72 \pm 2.4$ & $75 \pm 3.6$ & $79 \pm 3.0$ \\
Diphthong duration /aI/, ms & control & $178 \pm 69$ & $273 \pm 119$ & $231 \pm 92$ \\
& PD & $165 \pm 51$ & $224 \pm 99$ & $188 \pm 69$ \\
Diphthong duration /JI/, ms & control & $190 \pm 43$ & $304 \pm 80$ & $253 \pm 65$ \\
& PD & $178 \pm 53$ & $249 \pm 101$ & $206 \pm 52$ \\
Intelligibility & control & $0.32 \pm 0.07$ & $0.23 \pm 0.06$ & $0.22 \pm 0.09$ \\
& PD & $0.50 \pm 0.13$ & $0.38 \pm 0.15$ & $0.35 \pm 0.12$
\end{tabular}

Smaller numerical values for intelligibility (i.e., values closer to 0 ) indicate relatively better intelligibility.

peak amplitude using Goldwave version 5 [23], and then mixed with 20 -person babble $[24,25]$ in Goldwave. A signal-to-noise ratio of $-3 \mathrm{~dB}$ was applied to each sentence, as pilot testing indicated that this signal-to-noise ratio minimized both floor and ceiling effects. This signal-to-noise ratio also has been used in other studies [26,27]. Stimuli were presented to listeners at $75 \mathrm{~dB}$ SPL via headphones (Sony, MDR V300) in a double-walled audiometric booth.

Listeners judged intelligibility using a computerized, continuous $150-\mathrm{mm}$ visual analog scale [17]. Each sentence was judged without knowledge of the speaker's neurological diagnosis or identity. Written and verbal instructions directed listeners to judge how well sentences could be understood, with endpoints of the continuous scale labeled as 'Understand everything' to 'Cannot understand anything'. Sentences for all speakers from the larger database and all conditions were pooled and divided into 10 sets. Sets were constructed to ensure each set was comprised of one sentence produced by each talker in each condition and that all 25 Harvard Sentences were represented in similar proportions. Five listeners judged each set. To determine intrajudge reliability, $10 \%$ of the sentences were presented twice. Pearson product correlations for intrajudge reliability ranged from 0.60 to 0.88 (mean $=$ $0.72 ; \mathrm{SD}=0.07$ ) for the 50 listeners. Interjudge reliability was assessed using the intraclass correlation coefficient. Mean intraclass correlation coefficients ranged from 0.85 to 0.91 ( mean $=0.85$; $\mathrm{SD}=0.02)$ across the 10 sets and were significant $(\mathrm{p}<0.001)$. Listener reliability is considered further in the 'Discussion'.

\section{Data Analyses}

Descriptive and parametric statistics were employed. The variables of articulatory rate, SPL, average F2 slope, maximum F2 slope, and intelligibility were fit with a mixed linear model in this repeated measures design. The within-subjects factor was Condition (Habitual, Clear, Loud) and the between-subjects factor was Group (PD, Control). Covariates included age and speaker sex. F2 slope analyses also included diphthong segment duration as a covariate, given studies reporting a relationship between speech duration and F2 slope [2]. Post hoc comparisons were made using a Bonferroni correction. Relationships among variables were examined using correlation and regression analyses.

Consequences of Clear and Loud Speech

\section{Results}

\section{Speech Durations and Vocal Intensity}

Table 2 reports descriptive statistics for SPL and duration. The Condition effect was significant for articulation rate $[\mathrm{F}(2,52)=70.88, \mathrm{p}<0.0001]$ and $\operatorname{SPL}[\mathrm{F}(2,52)=$ $157.36, \mathrm{p}<0.0001]$. Post hoc testing within groups confirmed differences for all pairs of conditions ( $p<0.003)$, with the exception of the PD group's Habitual-Loud contrast for articulation rate. The Group effect also was significant for articulation rate $[\mathrm{F}(1,24)=9.39, \mathrm{p}=0.005]$ and mean SPL $[F(1,24)=10.76, \mathrm{p}=0.003]$. The interaction of Group and Condition also was significant for SPL $[F(2,52)=6.49, \mathrm{p}=0.003]$. Post hoc testing indicated that the interaction was due to the PD group having a reduced SPL compared to controls in both the Clear and Loud conditions, but not Habitual ( $p<0.004)$. Segment duration results were identical to those for articulation rate. To summarize, both groups increased vocal intensity in the Clear and Loud conditions relative to Habitual, with the magnitude of the increase being greatest for the Loud condition. SPL for the PD group also was reduced compared to controls in both the Clear and Loud conditions. Both groups also typically lengthened speech durations for the Clear and Loud conditions, although speech durations for the PD group tended to be accelerated compared to controls.

\section{F2 Slope}

Table 3 reports descriptive statistics for slope. Within groups and conditions, average and maximum slope metrics were significantly correlated for both diphthongs, with Pearson $r$ coefficients ranging from 0.76 to 0.92 
Table 3. Group means and SDs for F2 slope metrics ( $\mathrm{Hz} / \mathrm{ms})$

\begin{tabular}{llrrr}
\hline Measure & Group & Habitual & \multicolumn{1}{l}{ Clear } & \multicolumn{1}{l}{ Loud } \\
\hline Maximum F2 /aI/ & CF & $16.8 \pm 3.7$ & $17.3 \pm 4.9$ & $16.7 \pm 3.2$ \\
& PDF & $13.0 \pm 2.6$ & $14.3 \pm 2.9$ & $12.9 \pm 3.1$ \\
& CM & $10.7 \pm 1.3$ & $11.9 \pm 1.3$ & $11.1 \pm 1.3$ \\
& PDM & $9.8 \pm 1.9$ & $11.1 \pm 1.9$ & $10.0 \pm 1.6$ \\
Average F2 /aI/ & CF & $8.8 \pm 1.2$ & $8.5 \pm 1.5$ & $7.8 \pm 0.9$ \\
& PDF & $6.6 \pm 1.3$ & $6.9 \pm 1.0$ & $6.7 \pm 1.0$ \\
& CM & $6.6 \pm 0.8$ & $6.6 \pm 0.6$ & $6.4 \pm 0.7$ \\
Maximum F2 /JI/ & CFM & $5.6 \pm 1.0$ & $6.2 \pm 0.9$ & $5.8 \pm 1.2$ \\
& PDF & $23.4 \pm 6.3$ & $23.0 \pm 5.0$ & $22.8 \pm 7.5$ \\
& CM & $15.8 \pm 6.1$ & $17.7 \pm 5.9$ & $15.2 \pm 4.0$ \\
& PDM & $11.8 \pm 2.5$ & $12.9 \pm 3.2$ & $11.5 \pm 2.3$ \\
Average F2 /oI/ & CF & $10.3 \pm 1.9$ & $10.3 \pm 2.0$ & $9.6 \pm 2.1$ \\
& PDF & $7.5 \pm 1.7$ & $7.8 \pm 2.4$ & $7.7 \pm 1.5$ \\
& CM & $7.2 \pm 1.1$ & $7.3 \pm 1.1$ & $6.7 \pm 1.0$ \\
& PDM & $5.9 \pm 1.0$ & $6.6 \pm 1.2$ & $5.8 \pm 1.0$ \\
\hline
\end{tabular}

Speaker sex is denoted in Group codes ( $\mathrm{F}=$ females; $\mathrm{M}=$ males $)$.

(two-tailed tests, $\mathrm{p}<0.002$ ). Pooling data across groups and conditions yielded Pearson $\mathrm{r}$ coefficients for /oi/ and /aI/ of 0.90 and 0.88 , respectively. The Group effect was only significant for average slope metrics [/or/: $\mathrm{F}(1,25)=$ $16.70, \mathrm{p}<0.001 ; / \mathrm{aI} / \mathrm{F}(1,25)=15.69, \mathrm{p}<0.001]$. The Condition effect was significant for all slope metrics, with the exception of maximum slope for /oi/ [average /or/: $\mathrm{F}(2,51)=9.05, \mathrm{p}=0.0004 ;$ average $/ \mathrm{a} / \mathrm{:} \mathrm{F}(2,51)=21.28$, $\mathrm{p}<0.0001 ;$ maximum /aI/: $\mathrm{F}(2,51)=7.97, \mathrm{p}=0.001]$. Post hoc tests within each group indicated steeper slopes for Clear versus both the Habitual and Loud conditions $(\mathrm{p}<0.05)$, although for each group there was one instance in which the Clear-Loud contrast was not significant. Given the strong correlation between the two slope metrics as well as the fact that average but not maximum F2 slope consistently distinguished among groups and conditions, correlation and regression analyses reported in the remainder of the article were performed using average slope measures.

\section{Relationship between F2 Slope and SPL}

Regression analysis was used to quantify the relationship between SPL and average F2 slope within and across conditions for each group and diphthong. Data for males and females was pooled as the variable representing speaker sex was not significant in the statistical analysis for SPL. Only the Control group's Clear regression for / oi/ as well as the Control group's across-condition regression for /oI/ were significant $(\mathrm{p}<0.05)$. Functions accounted for 3 and $8 \%$ of the variance, respectively, with a steeper slope associated with lower SPL.

\section{Intelligibility and Acoustic Predictors of Intelligibility}

Descriptive statistics for intelligibility are reported in table 2. Values closer to ' 0 ' indicate better intelligibility. The Group effect was significant, with poorer overall intelligibility for speakers with $\operatorname{PD}[\mathrm{F}(1,24)=13.84$, p < 0.001]. The Condition effect also was significant $[\mathrm{F}(2,52)=26.85, \mathrm{p}<0.0001]$. Post hoc tests further indicated better intelligibility in the Clear and Loud conditions for both groups versus Habitual $(\mathrm{p}<0.003)$, but no difference for Clear and Loud.

The relationship of average F2 slope to intelligibility for the PD group was examined using hierarchical regression analysis. Additional predictor variables included articulation rate and vocal intensity. Speaker sex was not significant in the intelligibility analysis previously summarized. Thus, as in other studies, data for men and women were pooled [2]. F2 slope values were averaged across diphthongs thus yielding a composite F2 slope metric similar to composite metrics for articulation rate, SPL and intelligibility. The model including only F2 slope accounted for $14 \%$ of the variance in intelligibility $[\mathrm{F}(1,37)=7.388, \mathrm{p}=0.01]$. Adding articulation rate and SPL accounted for an additional 5 and $6 \%$ of the variance, respectively, although these increases were not statistically significant. The final model including all three predictors accounted for $23 \%$ of the variance in intelligibility $[F(3,35)=4.675, p=0.008]$. The sign of the standardized beta coefficients indicated that a steeper F2 slope, slower articulation rate and higher SPL were associated with better intelligibility. Finally, all variance inflation factors were less than 2.0, indicating the absence of serious multicollinearity among predictor variables.

\section{Discussion}

Major findings may be summarized as follows. Average and maximum F2 slope metrics were strongly correlated, but only average slope measures distinguished the two speaker groups, with shallower slopes for the PD group. With one exception, there also were significant differences in F2 slope metrics among speaking conditions, with steeper slopes for the Clear condition versus the Habitual and Loud conditions. Clear and Loud speech were further characterized by lengthened durations, increased intensity and improved intelligibility versus Ha- 
bitual. F2 slope and intensity were unrelated but F2 slope was a significant predictor of intelligibility. The remainder of the 'Discussion' considers these findings and their implications in more detail.

In addition to eliciting adjustments in segmental articulation, as inferred from $\mathrm{F} 2$ slope, it is worth reiterating at the outset that the magnitude production paradigm was successful in eliciting variations in vocal intensity, speech duration and intelligibility. The nature of changes in vocal intensity and speech duration were similar for the Clear and Loud conditions relative to Habitual, although the magnitude of the adjustments differed. The finding of similar magnitudes of improvement in intelligibility for the Clear and Loud conditions versus Habitual further suggests the feasibility of using either a clear speech style or an increased vocal intensity therapeutically to enhance intelligibility for speakers with mild dysarthria secondary to PD. Comparative studies employing training paradigms are needed to build upon these results to determine whether the short-term effects for experimental speech stimuli demonstrated here can be maintained in the long term for functional speech tasks.

The sensitivity of F2 slope to articulatory changes elicited by different global dysarthria therapy techniques was one topic of interest. With the exception of maximum F2 slope for /oI/, the Clear condition generally yielded F2 slopes for the PD group that not only were significantly different from Habitual, but also more closely approximated Habitual slopes for neurologically normal talkers. Thus, results support the suggestion that F2 slope might serve as an objective metric of treatmentrelated changes in the articulatory mechanism in PD [1], at least for behavioral techniques such as clear speech which focus on articulatory behavior. The finding that F2 slope metrics in the Loud condition did not differ from Habitual is not entirely unexpected given other studies $[9,10]$. However, it is important to note that an increased vocal intensity has been shown to be associated with other types of segmental articulatory adjustments. For example, an expanded vowel space area and enhanced spectral distinctiveness for stops as well as increased movement velocities and displacements may accompany an increased vocal intensity [e.g., 9, 10, 13, 26]. Thus, F2 slope may simply not be sensitive to intensityrelated adjustments in articulatory behavior. The lack of a robust relationship between F2 slope and SPL supports this suggestion.

The nature of the relationship between maximum and average F2 slope metrics also was of interest as well as the ability of both metrics to distinguish speakers with mild dysarthria from healthy controls. Average but not maximum F2 slope metrics were significantly reduced for the PD group versus controls. Thus, results do not support the suggestion that measures of extreme F2 slope are more sensitive to mild dysarthria than average slope measures [8]. The correlation analysis further indicated a great deal of redundancy or overlap for the two slope measures. Given that extreme F2 slope metrics were not consistently sensitive to condition-related adjustments in segmental articulation, the strong correlation between the two slope metrics, and the fact that only average F2 slope distinguished the PD and control groups, the value of obtaining maximum F2 slope metrics in dysarthria seems questionable. Before drawing strong conclusions, however, studies with greater speaker numbers and more varied phonetic contexts are needed.

Finally, an ongoing goal of dysarthria research is to identify aspects of speech potentially related to intelligibility. In the present study, a composite metric of average F2 slope was a significant predictor of intelligibility for the PD group when data were pooled across conditions, with articulation rate and vocal intensity explaining an additional small amount of the variance in intelligibility. Thus, as suggested in other studies, F2 slope is linked to intelligibility even in mild dysarthria and holds promise as an index of functional communication skill $[2,5]$. The final regression model indicated that steeper F2 slopes, slower articulation rates, and higher vocal intensities were associated with better intelligibility. A clinical implication is that therapeutic techniques that elicit an increase in F2 slope, reduced articulation rate and increased vocal intensity would likely also maximize intelligibility.

\section{Caveats}

Several factors should be kept in mind when interpreting results. The importance of studying intelligibility in dysarthria in adverse listening conditions has been noted, and multitalker babble is arguably an ecologically valid perceptual environment [11]. However, results may differ for speech in quiet. Listener reliability metrics further suggest the challenging nature of the intelligibility task. Although some reliability metrics may appear modest, our metrics compare well to other studies using scaling tasks to measure intelligibility in dysarthria [e.g., 28-30]. Moreover, when the intelligibility data were reanalyzed using only listeners with intrajudge reliability of $r=0.7$ or better, results were identical to those for the larger pool of 50 listeners. Finally, although unlikely, it is possible that LSVT history for 2 of 
the PD speakers had some bearing on the results. The direction of the effect would be to magnify differences between the Loud condition and other conditions, however. Thus, if anything, acoustic and intelligibility changes associated with the Loud condition are overestimated in the current study.

\section{Conclusions}

In conclusion, average diphthong F2 slope was more sensitive than maximum F2 slope for capturing articulatory involvement in mild dysarthria in PD. Future studies are needed to determine whether F2 slope metrics might be sensitive to disease presence in the articu- latory mechanism in PD prior to any observable reduction in intelligibility. F2 slope also holds promise as an objective measure of treatment-related changes in the articulatory mechanism for therapeutic techniques that focus on eliciting changes in articulation. Studies employing kinematic, acoustic, and perceptual methods, such as that of Yunusova et al. [2], would aid in further understanding the nature of the speech movements responsible for changes in F2 slope and the impact on intelligibility.

\section{Acknowledgments}

Research was supported by R01DC004689.

\section{References}

1 Weismer G, Yunusova Y, Bunton K: Measures to evaluate the effects of DBS on speech production. J Neurolinguist 2012;25:74-94.

-2 Yunusova Y, Green JR, Greenwood L, Wang J, Pattee GL, Zinman L: Tongue movements and their acoustic consequences in amyotrophic lateral sclerosis. Folia Phoniatr Logop 2012;64:94-102.

3 Weismer G, Kim Y: Classification and taxonomy of motor speech disorders: what are the issues; in Maassen B, van Lieshout PHHM (eds): Speech Motor Control: New Developments in Basic and Applied Research. Oxford, Oxford University Press, 2010, pp 229-241.

4 Kim YJ, Weismer G, Kent RD, Duffy JR: Statistical models of $\mathrm{F} 2$ slope in relation to severity of dysarthria. Folia Phoniatr Logop 2009; 61:329-335.

5 Kim YJ, Kent RD, Weismer G: An acoustic study of the relationships among neurologic disease, dysarthria type and severity of dysarthria. J Speech Lang Hear Res 2011;54:417-429.

6 6 Weismer G, Kent RD, Hodge M, Martin R: The acoustic signature for intelligibility test words. J Acoust Soc Am 1988;84:1281-1291.

7 Richards E, Tjaden K, Lam J, Wilding G: Diphthong F2 trajectories in dysarthria: clear, loud and slow speech. ASHA Convention, Philadelphia, 2010.

8 Rosen KM, Goozée JV, Murdoch BE: Examining the effects of multiple sclerosis on speech production: does phonetic structure matter? J Commun Disord 2008;41:49-69.

9 Dromey C, Ramig LO, Johnson AB: Phonatory and articulatory changes associated with increased vocal intensity in Parkinson disease: a case study. J Speech Hear Res 1995;38:751-764.

10 Tjaden K, Wilding GE: Rate and loudness manipulations in dysarthria: acoustic and perceptual findings. J Speech Lang Hear Res 2004;47:766-783.
11 Yorkston KM, Hakel M, Beukelman DR, Fager S: Evidence for effectiveness of treatment of loudness, rate, or prosody in dysarthria: a systematic review. J Med Speech Lang Pathol 2007; 15:xi-xxxvi.

-12 Fox CM, Morrison CE, Ramig LO, Sapir S: Current perspectives on the Lee Silverman Voice Treatment (LSVT) for individuals with idiopathic Parkinson disease. Am J Speech Lang Pathol 2002;11:111-123.

13 Sapir S, Spielman J, Ramig L, Story B, Fox C: Effects of intensive voice treatment (the Lee Silverman Voice Treatment [LSVT]) on vowel articulation in dysarthric individuals with idiopathic Parkinson's disease: acoustic and perceptual findings. J Speech Lang Hear Res 2007; 50:899-912.

14 Cookman S, Verdolini K: Interrelation of mandibular laryngeal functions. J Voice 1999; 13:11-24.

15 Sapir S: The intrinsic pitch of vowels: theoretical, physiological, and clinical considerations. J Voice 1989;3:44-51.

16 YorkstonKM,BeukelmanDR,StrandEA,Hakel M: Management of Motor Speech Disorders in Children and Adults. Austin, PRO-ED, 2010.

17 Sussman JE, Tjaden K: Perceptual measures of speech from individuals with Parkinson's disease and multiple sclerosis: intelligibility and beyond. J Speech Lang Hear Res 2012;55: 1208-1219.

18 Molloy D: Standardized Mini-Mental State Examination. Troy, New Grange Press, 1999.

19 Yorkston K, Beukelman D: Sentence Intelligibility Test for Windows. Lincoln, Tice Technologies, 1996.
20 IEEE: IEEE Recommended Practice for Speech Quality Measurements. Institute of Electrical and Electronic Engineers, New York, 1969.

21 Milenkovic P: TF32 [computer software]. Madison, University of Wisconsin-Madison, 2005.

22 Smiljanić R, Bradlow AR: Speaking and hearing clearly: talker and listener factors in speaking style changes. Lang Linguist Compass 2009;3:236-264.

23 Goldwave Inc: Goldwave (version 5) [software]. http://www.goldwave.com/release.php, 2010.

24 Frank T, Craig CH: Comparison of the Auditec and Rintelmann recordings of the NU-6. J Speech Hear Disord 1984;49:267-271.

25 Nilsson M, Soli S, Sullivan J: Development of the hearing in noise test for the measurement of speech reception thresholds in quiet and in noise. J Acoust Soc Am 1994;95:1085-1099.

26 Ferguson S, Kewley-Port D: Vowel intelligibility in clear and conversational speech for normal-hearing and hearing-impaired listeners. J Acoust Soc Am 2002;112:259-271.

27 Maniwa K, Jongman A, Wade T: Perception of clear English fricatives by normal-hearing and simulated hearing-impaired listeners. J Acoust Soc Am 2008;123:1114-1125.

28 Neel AT: Effects of loud and amplified speech on sentence and word intelligibility in Parkinson disease. J Speech Hear Res 2009;52:10211033.

29 Van Nuffelen G, De Bodt M, Wuyts F, Van de Heyning P: The effect of rate control on speech rate and intelligibility in dysarthria. Folia Phoniatr Logop 2009;61:69-75.

30 Yunusova Y, Weismer G, Kent RD, Rusche NM: Breath-group intelligibility in dysarthria: characteristics and underlying correlates. J Speech Hear Res 2005;48:1294-1310. 